\title{
Neutrino Target of Opportunity program for the Cherenkov Telescope Array
}

\author{
Konstancja Satalecka*1, Anthony M. Brown ${ }^{2}$, Alberto Rosales de Leon ${ }^{2}$, Olga \\ Sergijenko ${ }^{3}$ for the CTA Consortium ${ }^{\dagger}$ \\ ${ }^{1}$ DESY, 15738 Zeuthen, Germany, ${ }^{2}$ Centre for Advanced Instrumentation (CfAI), Department of \\ Physics, University of Durham, Durham, UK, ${ }^{3}$ Astronomical Observatory, Taras Shevchenko \\ National University of Kyiv, 04053 Kyiv Ukraine \\ E-mail: konstancja.satalecka@desy.de, anthony.brown@durham.ac.uk, \\ alberto.rosales-de-leon@durham.ac.uk, \\ olga.sergijenko.astro@gmail.com
}

\section{Chun Fai Tung ${ }^{4}$, René Reimann ${ }^{5}$, Theo Glauch ${ }^{6}$, Igancio Taboada ${ }^{4}$ for the FIRESONG Team \\ ${ }^{4}$ Center for Relativistic Astrophysics and School of Physics. Georgia Institute of Technology. Atlanta, GA 30332, USA., ${ }^{5}$ III. Physikalisches Institut, RWTH Aachen University, D-52056 Aachen, Germany, ${ }^{6}$ Institute for Advanced Studies, Technical University of Munich, D-85748 Garching, Germany \\ E-mail: ctung6@gatech.edu, reimann@physik.rwth-aachen.de, theo.glauchetum.de, itaboada@gatech.edu}

The measurement of an astrophysical flux of high-energy neutrinos by IceCube is an important step towards finding the long-sought sources of cosmic rays. Nevertheless, the long exposure neutrino sky map shows no significant indication of point sources so far. This may point to a large population of faint, steady sources or flaring objects as origins of this flux. The most compelling evidence for a neutrino point source so far is the recent observation of the flaring gamma-ray blazar TXS 0506+056 in coincidence with a high-energy neutrino from IceCube. This is a result of a Neutrino Target of Opportunity (NToO) program in which all currently operating Imaging Atmospheric Cherenkov Telescopes (IACTs) take part. The case for TXS 0506+056 being a neutrino source was made stronger by evidence of a 5-month long neutrino flare in 2014-2015. Here we investigate the chances of a detection of a gamma-ray counterpart to a neutrino source with CTA, as a result of a follow-up observation of a neutrino alert. We use the FIRESONG software to simulate different neutrino sources populations, which could be responsible for the diffuse flux of astrophysical neutrinos as measured by IceCube. We scan over parameters that can be used to describe the populations such as density (density rate) for steady (flaring) objects. Several CTA array layouts and instrument response functions are tested in order to derive optimal follow-up strategies and the potential science reach of the NToO program for CTA. We find that following neutrino alerts by IceCube, CTA has a low per alert probability of detecting a matching steady source. However, using a model by Halzen et al. (2018), for neutrino flares similar to that of 2014-2015, we find that CTA will detect a counterpart in as many as one third of the alerts.

36th International Cosmic Ray Conference -ICRC2019-

July 24th - August 1st, 2019

Madison, WI, U.S.A. 


\section{Introduction}

The Cherenkov Telescope Array will be the next generation ground-based imaging atmospheric Cherenkov telescope (IACT) array. Building upon the strengths of the current generation of telescopes, such as MAGIC, H.E.S.S. and VERITAS, CTA will detect gamma rays from $20 \mathrm{GeV}$ in energy, to above $300 \mathrm{TeV}$, with an order of magnitude improvement in sensitivity, as well as improved angular and energy resolution compared to the current generation IACTs. To achieve this improved performance CTA will consist of three telescope sizes, small, medium and large, (SST, MST and LST respectively), with each one optimised for a specific energy range. This size optimisation is defined by the physical processes governing photon-induced extended air showers (EAS), as the number of Cherenkov photons they emit increases with energy.

With a mirrored surface area of $370 \mathrm{~m}^{2}$, the LST is designed to observe the low flux of Cherenkov photons from EAS induced by $E_{\gamma}<200 \mathrm{GeV}$ photons. Given the relative abundance of $E_{\gamma}<200 \mathrm{GeV}$ photons, there will be a small number of LSTs at the center of each CTA array. With a mirror area of $\sim 88 \mathrm{~m}^{2}$, the MSTs will be more numerous than the LSTs, and will provide the majority of CTA's sensitivity improvement in the $0.1 \leq E_{\gamma} \leq 10 \mathrm{TeV}$ photon energy range. With an effective mirror area of $\sim 8 \mathrm{~m}^{2}$, the SSTs will be sensitive to the highest energy photons CTA will observe, $E_{\gamma} \geq 5 \mathrm{TeV}$. This mirror area is sufficient since, at these photon energies, the limiting factor is the number of photon-induced EAS, not the flux of Cherenkov photons emitted by each EAS. As such, the SSTs will be the most numerous telescope design in CTA and will be spread over $4 \mathrm{~km}^{2}$. To achieve all-sky coverage, CTA will consist of two arrays, one in each hemisphere, with the northern array consisting of 19 telescopes spread over $1 \mathrm{~km}^{2}$ of the La Palma landscape, specifically the IAC, and the southern array consisting of 99 telescopes spread over $4 \mathrm{~km}^{2}$ of the Chilean landscape, on an ESO site between the ELT and VLT.

In the context of the origin of transient astrophysical messengers, like neutrinos, CTA has several important performance characteristics. Firstly, CTA's telescopes are designed to rapidly re-position to any location in the sky. As a point of reference, the LSTs can reposition to anywhere in the sky above $30^{\circ}$ in just 30 seconds, thus minimising the time between receiving alerts from other observatories, such as IceCube, and starting observations. Secondly, the MST and LSTs have a large field-of-view (FoV); the FoV for the SSTs will be $\sim 10^{\circ}$.

Astrophysical sources capable of hadronic acceleration to relativistic energies have long been believed to be sources of astrophysical neutrinos, with the neutrinos originating from the decay of charged mesons created by the relativistic hadrons interacting with ambient gas and/or ambient radiation within the astrophysical source. The decay of these mesons results in the emission of neutrinos and gamma rays. As such, assuming that the photon opacity of the emission region is low enough to allow gamma rays to escape, searching for spatially and temporally correlated gamma-ray and neutrino emission allows us to constrain the origin of the astrophysical neutrinos observed by the IceCube Neutrino Observatory.

In April 2016, IceCube initiated a Realtime program in which neutrinos, with high probability of being of astrophysical origin, are reported in real time via the Gamma-ray-burst Coordinate Network (GCN) [1]. The program reports $v_{\mu}$ candidate events, which have the advantage of well-

\footnotetext{
* Speaker.

†https: / / www . cta-observatory . org/ For collaboration list, see PoS(ICRC2019) 1177.
} 
localized angular positions, typically $\Delta \psi \sim 0.5^{\circ}$. Starting on June 17th, 2019, a new version of the Realtime program became operational [2]. In this updated system, two streams of neutrinos are provided. The Gold stream corresponds to $\sim 10$ events per year with an average astrophysical signal purity of 50\%. The Bronze stream has an average purity of 30\% for an additional $\sim 20$ alerts per year.

In the CTA transient key science program (KSP) a total of 5 hours per site, per year, has been allocated to high-energy neutrino events observations, during the first 10 years of CTA operation. In these proceedings, we outline the ongoing work to quantify the efficiency with which CTA will observe gamma-ray emission from IceCube's 'Gold' $v_{\mu}$ alerts and all-sky 'hot-spots' (i.e. highest significance positions). The structure of the proceedings is as follows: in Section 2 we outline the simulation tool set we used to simulate IceCube's alerts, in Section 3 we outline our conversion from neutrino flux to expected gamma ray flux. Section 4 describes our simulations of CTA's response to the expected gamma-ray flux, while in Section 5 we state our results.

\section{FIRESONG}

The sources of astrophysical high-energy neutrinos have not been unequivocally identified. Observations of TXS 0506+056 provide evidence that blazars or a sub-population of blazars are responsible for the neutrino flux [3,4]. Nevertheless, evidence has only been claimed for one blazar/neutrino correlation therefore one has to be careful while extending its properties to describe the whole population of neutrino sources. This lack of knowledge has been parametrized in terms of local density (local density rate) vs. neutrino luminosity (flare energy) for steady (flaring) sources [5].

FIRESONG ${ }^{1}$ is a freely available software that simulates a neutrino population, given these two parameters, while making additional assumptions on source evolution, e.g. that source density scales with star formation rate (SFR), and luminosity functions, e.g. that neutrino sources are standard candles with constant neutrino luminosity. In these proceedings we explore a source population which does not evolve with redshift (which is a simplified way to describe blazars) and one that follows the SFR. Both are described as standard candles. The software allows for other alternatives. We also assume that the specific class simulated is responsible for $100 \%$ of the astrophysical neutrino flux [6]. FIRESONG is able to describe alerts with the same characteristics as those reported by IceCube. It should be noted, that the rate and declination dependence of alerts depend on IceCube's response, notably the effective area. However, the redshift distribution of the sources of alerts depends on the cosmology model and the properties of the assumed population.

\subsection{TXS-like flaring sources}

The first scenario simulated is modeled after the neutrino flare of TXS 0506+056 (TXS) in the season 2014-2015. We follow the model suggested by [7] in which only a fraction of blazars, including TXS, are responsible for the astrophysical neutrino flux. We will call this population TXS-like sources. The local burst density rate can be related to the local density of blazars as:

$$
\dot{\rho}=F \times \rho_{\mathrm{BL}} .
$$

\footnotetext{
${ }^{1}$ https://github.com/ChrisCFTung/FIRESONG
} 
where $F$ is a fraction of the total number of blazars. As described before, we assume these sources are neutrino standard candles and display no density rate evolution. The flare distribution as a function of redshift is shown in Figure 1a. We also assume all the TXS-like sources have the same flare duration in their local reference frame. Correcting the 110 days flare of TXS [3] for redshift, this corresponds to 82 days. Saturating the astrophysical neutrino flux with these flares results in a uniquely defined burst energy as a function of $F$. The neutrino flux at Earth as a function of redshift for a fixed $F$ is shown in Figure $1 b$.

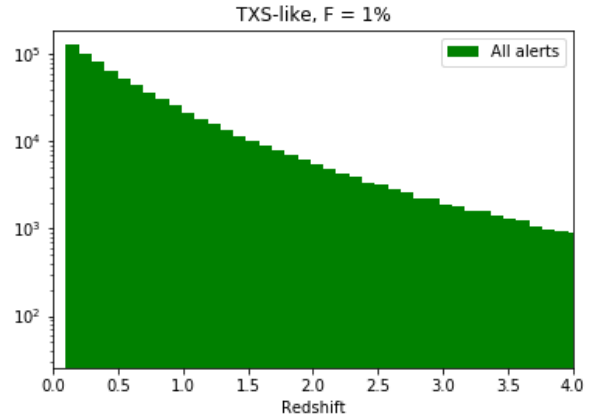

(a)

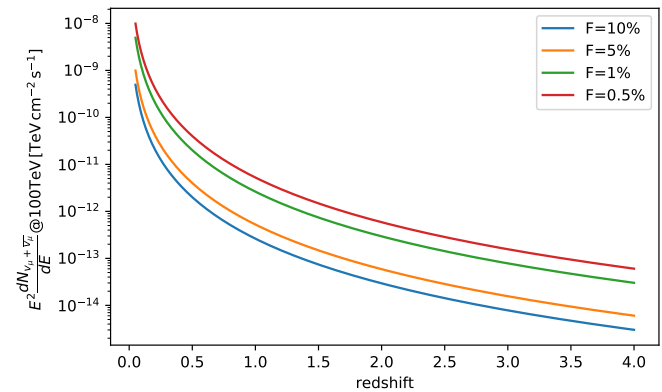

(b)

Figure 1: Simulated neutrino sources parameters for "TXS-like" flaring blazars. Left: redshift distribution of the simulated alerts for flaring sources fraction $F=1 \%$. Right: neutrino flux at 100 $\mathrm{TeV}$ as a function of the redshift, for the four flaring sources fractions $F$.

\subsection{Steady sources}

The second scenario simulated is is that the diffuse neutrino flux is due to steady neutrino sources. Although IceCube has not yet resolved a point source above $5 \sigma$ significance, there are sources that have exceeded the IceCube sensitivity (Note that sensitivity in IceCube has a different definition than for IACTs; see Ref. [8]). These sources will be of interest for the NToO program of CTA. Assuming the neutrino sources are i) standard candles, ii) follow the star formation evolution from [9], and iii) saturate the astrophysical neutrino flux [6], we can simulate the whole-sky source population with different local density conditions. Figure 2 a shows the resultant distribution of the neutrino fluxes at Earth under the condition that local density $=3 \times 10^{-9} \mathrm{Mpc}^{-3}$. Then, the flux from each neutrino source is compared with IceCube's sensitivity [8]. The sources that exceed IceCube's sensitivity are used as seeds of the NToO for CTA. The distribution of redshift before and after applying the sensitivity selection is shown in Figure $2 \mathrm{~b}$.

\section{VHE gamma-ray emission accompanying the neutrino emission}

In order to calculate the gamma-ray flux emitted together with neutrinos we assume that they are produced in proton interactions with the surrounding photon ( $p \gamma$ interactions) or matter fields ( $p p$ interactions). The secondary pions and other particles decay to neutrinos or gamma rays and in the simplest case the relation between the gamma ray and neutrino production rates is:

$$
\frac{1}{3} \sum_{\alpha} E_{v}^{2} A_{v_{\alpha}}\left(E_{v}\right)=\frac{K_{\pi}}{4} E_{\gamma}^{2} A_{\gamma}\left(E_{\gamma}\right)
$$




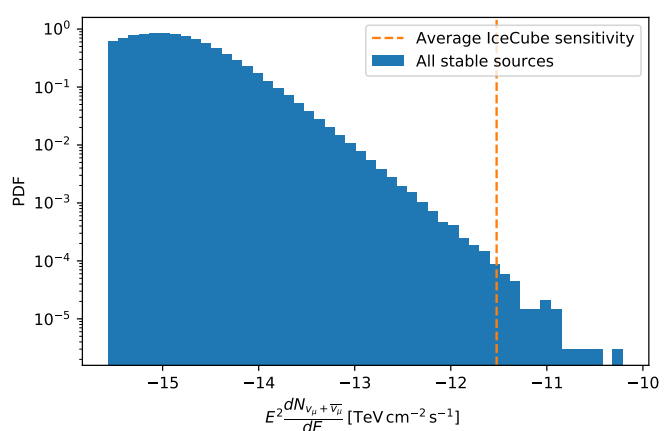

(a)

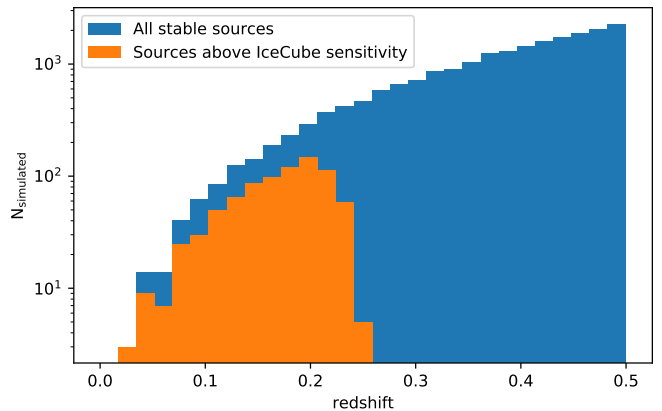

(b)

Figure 2: Simulated steady neutrino sources parameters for sources following SFR evolution with local density $3 \times 10^{-9} \mathrm{Mpc}^{-3}$. Left: distribution of the neutrino flux at $100 \mathrm{TeV}$. The northern-sky declination-averaged sensitivity of IceCube is shown by the orange broken line. Right: redshift distribution.

where $\mathrm{E}_{\gamma}=2 \mathrm{E}_{v}$ and $\mathrm{K}_{\pi}$ is a factor which accounts for the ratio of charged to neutral pions: $\mathrm{K}_{\pi}=$ 2 for $p p$ and $\mathrm{K}_{\pi}=1$ for $p \gamma$ interactions (for full derivation see e.g. [10]). In the case of steady sources we assume $p \gamma$ interactions, as usually postulated for Active Galactic Nuclei (AGN), and do not consider any additional absorption or cascading of $\gamma$ rays inside the source.

In the case of TXS-like sources we adapt the phenomenological model of [7]. The emerging gamma-ray flux is given by:

$$
\frac{d N_{\gamma}}{d E}=A_{v} E^{-2} e^{\left(-E_{L} / E-E / E_{H}\right)}
$$

where $\mathrm{E}_{L}$ and $\mathrm{E}_{H}$ are low- and high-energy cutoffs, and $\mathrm{A}_{v}$ is the simulated neutrino flux normalization. In a case of TXS 0506+056 located at redshift $z=0.335$, the $\mathrm{E}_{L}=0.1 \mathrm{TeV}$ and $\mathrm{E}_{H}=20$ $\mathrm{TeV}$ (see [7]). For for sources located at different redshifts, we scale those values accordingly.

\section{CTA simulations}

To simulate the CTA follow-up observations of the neutrino alerts we use the ctools-1.5.4 package with gammalib-1.5.4 [11]. We employ the prod3b-v2 CTA instrument response functions (IRFs) with zenith angles $20^{\circ}, 40^{\circ}, 60^{\circ}$ and azimuth dependence following the different magnetic field configurations at each site.

We obtain the redshift, spectrum normalization and declination for the alerts from running FIRESONG, while the right ascension is assigned randomly. For all sources, we take into account extragalactic background light (EBL) absorption [12]. EBL cutoffs of hundreds of GeV are expected for many targets predicted to be neutrino sources with FIRESONG simulations, which means that the prospects for detection depend on the low energy threshold of CTA (see Figure 3). We take into account the energy dispersion of CTA.

For each alert, we simulate the photon events list for $10 \mathrm{~min}$ of either North or South Baseline Array (BA) observations with a $5.0^{\circ} \mathrm{ROI}$ centered at a source (with the tool ctobsssim). We consider the following energy ranges: $0.02-200 \mathrm{TeV}$ for $20^{\circ}, 0.025-200 \mathrm{TeV}$ for $40^{\circ}$ and $0.1-$ $200 \mathrm{TeV}$ for $60^{\circ}$ zenith angles (the lower and upper limits are defined by the IRFs). For these 
observations we then perform a maximum likelihood fitting using the tool ct like in an unbinned mode. A test statistic (TS) equal or higher than 25 qualifies as a source detection at the $\sim 5 \sigma$ level.
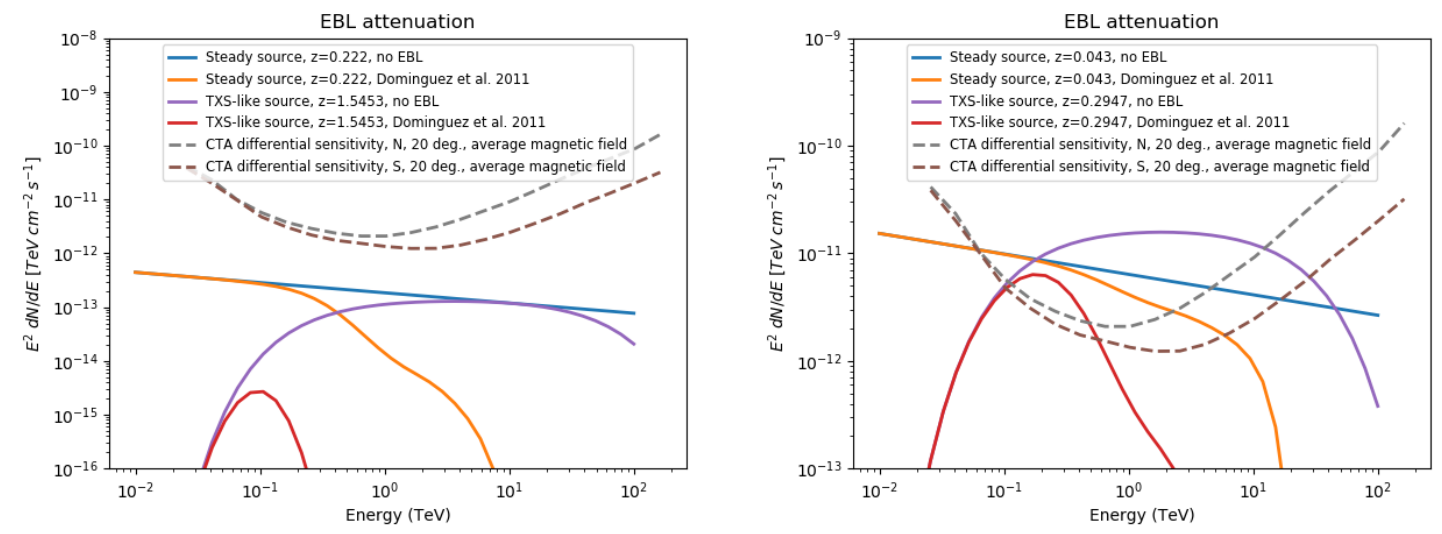

Figure 3: The energy spectra without and with the EBL attenuation vs the CTA differential sensitivity for the undetected (left) and detected (right) sources.

\section{Results}

Figure 4a presents the detection probability of a "TXS-like" flaring neutrino source by CTA depending on the flaring source fraction $F$ and the array configuration. The results obtained for zenith angles $20^{\circ}, 40^{\circ}, 60^{\circ}$ were stacked together with a weight of $1 / 3$ each. The shaded area represents the systematic uncertainty due to the magnetic field configuration at each site. For the lowest flaring source fractions, the flux of each flare and hence the detection probability is greatest. In the most optimistic case of $F=0.5 \%$ CTA has a $~ 30 \%$ chance of detection, assuming that $50 \%$ of the IC alerts correspond to real sources and all of them are observable by CTA. It should be noted that the limited CTA duty cycle of 10-15\% will prevent the fast follow-up of all neutrino alerts. This limitation is relevant for transients with activity timescales that are below several months, which we have not considered here.

In Fig.4b the redshift distribution of the generated and detected "TXS-like" alerts is shown for an example CTA configuration (Northern array with average magnetic field) and flaring sources fraction of $1 \%$. A clear cut-off at $z=0.6$ is present for zenith angles of $20^{\circ}$. As expected for higher zenith angles, the reach of CTA is limited to lower redshifts. The cut-off shifts with $F$ reaching 0.8 , 0.4 and below 0.4 for $F$ of $0.5 \%, 5 \%$ and $10 \%$ respectively.

The probability to detect a steady neutrino source as a function of the local source density is shown in Fig. 5a. The shaded region represents the uncertainty due to statistical fluctuations and the magnetic field configuration at each site. The detection probability increases with source density due to the IceCube's selection. As shown in Fig. 2a, the flux normalization decreases with increasing source density so that the neutrino flux is fully saturated by the simulated population. IceCube is more likely to detect an object emitting higher flux, therefore the distribution of the sources which would produce an alert in IceCube is biased to higher fluxes and lower redshifts as the density increases. As a consequence, the CTA chances at detection after receiving an alert 
from IceCube are higher. In all cases the detection probability is lower than for "TXS-like" flares, not surprising considering the fact that the per-source flux normalization is lower and the shortness of the simulated observation time $(10 \mathrm{~min})$. Due to difficulties in reaching an exact estimate, the IceCube background "hot-spot" fraction was not taken into account here. CTA duty cycle limitations are of less concern in a search for steady counterparts.

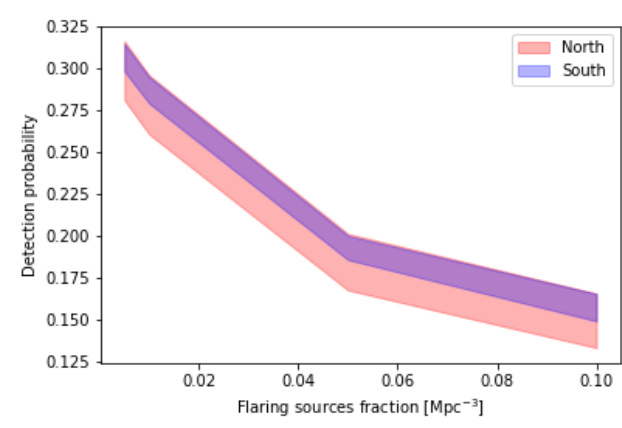

(a)

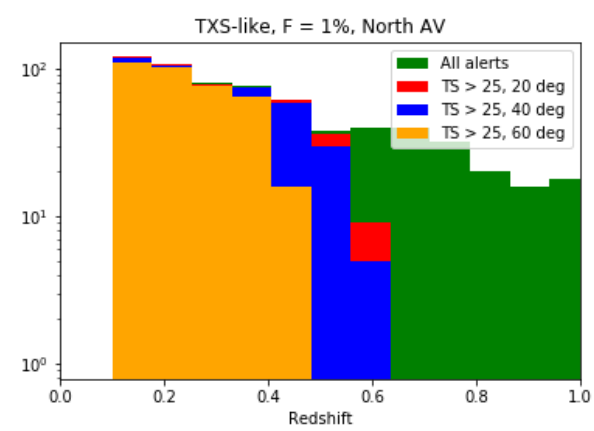

(b)

Figure 4: Results of CTA simulations for "TXS-like" sources. Left: probability to detect a neutrino source as a function of the flaring sources fraction $F$. The bands show the systematic uncertainty due to different magnetic field configurations at the site. Right: redshift distribution of the simulated and detected (North array, average magnetic field) alerts, flaring sources fraction $F=1 \%$.

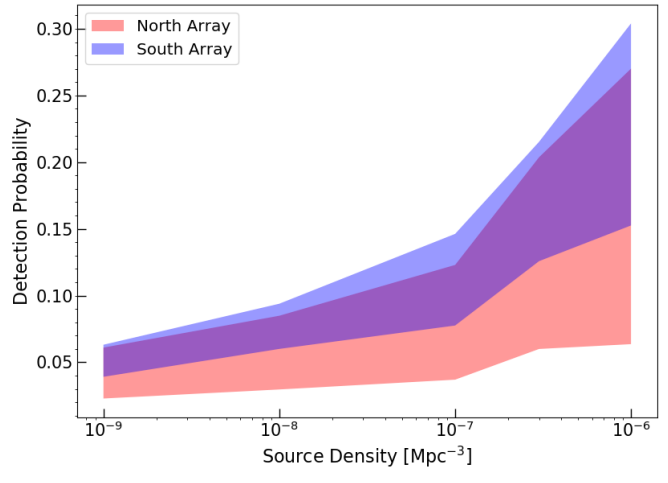

(a)

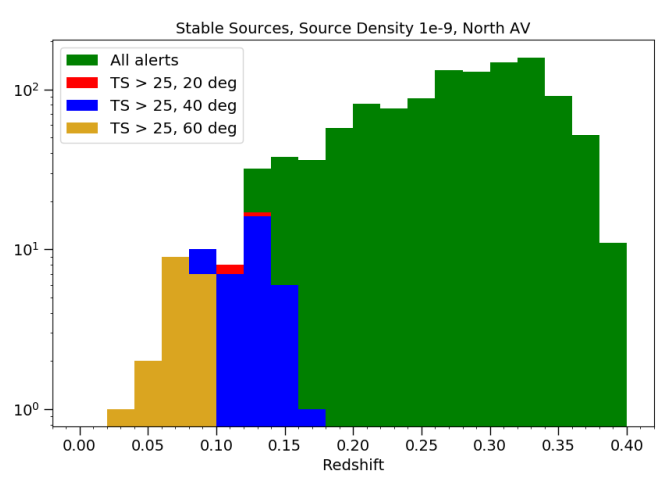

(b)

Figure 5: Results of CTA simulations for steady sources following a SFR evolution. Left: probability to detect a neutrino source as a function of the local source density. The uncertainty due to statistical fluctuations is shown like a shaded region. Right: redshift distribution of the simulated and detected (North array, average magnetic field) alerts, local source density $10^{-9} \mathrm{Mpc}^{-3}$

\section{Outlook}

In these proceedings we investigate the chances of CTA, the next generation IACT array to detect $\gamma$-ray signal after following an IceCube alert. Using the FIRESONG software package we simulate steady and flaring "TXS-like" neutrino source populations. We assume that the simulated 
neutrino source population is responsible for $100 \%$ of the neutrino flux. Only hadronic contributions to the $\gamma$-ray emission have been included. We simulate the CTA response with the ctools package and most recent instrument response functions for different array configurations, assuming 10 min observations per alert.

In these proceedings we test only a limited number of possible scenarios. Within those, CTA prospects are particularly promising for neutrino sources similar to the 2014-1015 neutrino flare by TXS 0506+056 as modeled by Halzen et al. [7].

In an upcoming study we plan to simulate neutrino emitting transients of different duration (e.g. $100-10000 \mathrm{~s}$ ) and a realistic CTA response, including e.g. delays due to alert distribution time and telescope repositioning. Different redshift evolution and source emission models will be tested. In the case of stable sources it is worth to investigate also longer observation times, which are still compatible with the KSP observation plan.

\section{Acknowledgment}

$\mathrm{KS}, \mathrm{AB}, \mathrm{ARdL}$ and OS gratefully acknowledge financial support from the agencies and organizations listed here: www.cta-observatory.org/consortium_acknowledgments. This work was performed within the CTA Transients Physics Working Group. CFT and IT acknowledge support by NSF grant PHY-1505230. ARdL acknowledges the support of the National Council for Science and Technology from Mexico (CONACYT).

This research made use of ctools, a community-developed analysis package for Imaging Air Cherenkov Telescope data. ctools is based on GammaLib, a community-developed toolbox for the scientific analysis of astronomical gamma-ray data.

\section{References}

[1] M.G. Aarsten, et al. (IceCube Collaboration). Astropart. Phys. 92 (2017) 30

[2] E. Blauffus, T. Kinstcher, L. Lu and C.F. Tung for the IceCube collaboration. These proceedings, PoS(ICRC2019)1021.

[3] M. G. Aartsen et al. [IceCube Collaboration], Science 361, no. 6398, 147 (2018)

[4] M. G. Aartsen et al. [IceCube Collaboration], Science 361, no. 6398, 150 (2018)

[5] M. Kowalski. Proceedings of the Eur. Cosmic Ray Symp. 2014. arXiv:1411.4385

[6] M. G. Aartsen et al. [IceCube Collaboration], Proceedings of ICRC 2017. arXiv:1710.01191 [astro-ph.HE].

[7] F. Halzen, A. Kheirandish and T. Weisgarber and S.P. Wakely ApJ 874 (2019) L9

[8] M. G. Aartsen et al. [IceCube Collaboration], Eur. Phys. J. C 79, no. 3, 234 (2019)

[9] P. Madau and M. Dickinson, Ann. Rev. Astron. Astrophys. 52, 415 (2014)

[10] Ahlers M., Halzen F., 2018, PrPNP, 102, 73

[11] J. Knoedlseder et al., A \& A, 593, A1 (2016).

[12] A. Dominguez et al., Mon. Not. Roy. Astron. Soc., 410, 2556 (2011) 